\title{
Design and Implementation of Intelligent Agricultural System Based on Zigbee
}

\author{
Peini Shang \\ School of information engineering, Yulin University, Yulin, Shaanxi, China \\ 157724554@qq.com
}

\begin{abstract}
Based on WiFi and Zigbee technology, an intelligent agricultural system was designed and implemented. The main function of the system is to monitor and adjust the temperature, humidity, light intensity and soil moisture. The whole system consists of three modules, the data acquisition and control module by Zigbee wireless sensor network data acquisition node and the coordinator module, acquisition and regulation can complete the environmental parameters; data processing and communication module is realized by $\mathrm{S} 3 \mathrm{c} 2440$ and the $\mathrm{WiFi}$ module, it can communicate with the coordinator module through the serial port, and through WiFi communicate with the Android control module; wireless remote control module served by Android intelligent equipment, through the monitoring program, can achieve real-time detection and control of environmental parameters. The test results show that the system performs well and runs stably.
\end{abstract}

Keywords: Zigbee protocol stack; Intelligent agricultural system; Wireless sensor network; CC2530

\section{基于Zigbee的智慧农业系统设计与实现}

尚佩妮

(榆林学院 信息工程学院, 陕西 榆林 719000)

摘要: 基于 Wifi 和 Zigbee 技术完成了智慧农业系统的设计与实现, 系统设计完成后可以实现对农作物生产环境的温湿 度、光照度及土壤湿度等的监控及自动调节。整个系统由三个模块组成, 其中数据采集及控制模块由 Zigbee 无线传感网络 的数据采集节点和协调器模块组成, 可以完成环境参数的采集及调节; 数据处理及通信模块由 S3c 2440 及 Wifi 模块来实现, 通过串口与协调器模块通信, 通过 WIfI 与 Android 控制模块进行通信; 远程无线控制模块由 Android 智能设备担当, 通过 监控程序, 可以实现环境参数的实时检测及控制调节。测试结果表明, 系统表现良好, 运行稳定。

关键词: Zigbee 协议栈; 智慧农业系统; 无线传感网络; CC2530

中图分类号: TP272 文献标志码: A

引言

当前农业发展正处于从传统向现代化农业过渡的进程当中，急需用现代物质条件进行装备，用现代科 学技术进行改造, 用现代经营形式去推进, 用现代发展理念引领。[1]而物联网技术的快速发展, 将会为 我国农业发展与世界同步提供一个国际领先的全新的平台，物联网技术也称为传感网技术，是典型的具有 交叉学科性质的军民两用战略高科技。[2，3]它综合了传感器技术、嵌入式计算机技术、分布式信息处理 技术、现代网络及无线通信技术等，能够通过各类集成化的微型传感器协作地实施监测和采集各种被测对 象的信息, 如湿温度、水分、压力等, 这些信息通过无线方式被发送, 并以自组多跳的网络方式传送到用 户终端，从而实现物理世界、计算机世界以及人类社会的三元世界联通。 $[4 ， 5]$

所谓智慧农业温室大棚系统是指利用先进的物联网技术、计算机网络技术、音频技术、无线通信技术 以及专家知识与智慧等技术, 使农业系统更加聪明、更加有效、更加智慧的运转, 达到充分利用农村资源、 
提高农产品竞争力、和谐农村和保护环境的目标, 实现农业可视化远程监测、远程操作、远程专家指导等 职能管理。 $[6,7,8]$ 智慧农业温度大棚可以帮助农民及时发现问题, 并且准确地确定发生问题的位置, 使农业生产自动化、智慧化，并可远程控制，提高农业生产的管理效率、加快了智慧农业的建设步伐。[9]

本文利用 ZigBee 和 WiFi 的无线通讯技术，设计了一个智慧农业温室大棚系统。该系统就是一种智 慧农业的信息管理系统，分为两个方面：数据采集和远程控制。数据采集主要负责对温室大棚内环境参数 如温度、湿度等的采集, 并将这些参数通过远程无线网络发送至用户可视的互联网平台界面, 为农业综合 生态信息自动检测, 对环境进行自动控制和智慧化管理提供科学依据, 更好地带动农业科技的发展。[10, $11]$

\section{1 系统设计总体方案}

\section{1 系统总体功能}

智慧农业管理系统旨在实现的以下主要功能:

(1) 农作物生长环境参数的实时采集，如大棚温湿度、大棚光照强度、土壤湿度等;

(2) 对农作物生长环境进行实时必要的自动调节, 系统可以根据实际需要进行环境参数的设置, 根据 这些参数设置, 来进行环境参数的调节, 如利用风扇模块及大棚卷窝模块进行温湿度的调节, 利用补光设 备进行光照强度的调节，利用水泵对土壤进行自动灌溉等;

(3) 制定人机交互界面, 随着 Android 手机的普遍使用, 用户直接可以根据自己的需要自行调节各个 终端之间的参数。

\section{2 系统总体结构}

系统总体结构如图 1 所示, 无线传感网络模块由一个 Zigbee 协调器模块及多个终端节点组成, 每一 个终端节点都连接了土壤湿度传感器、温湿度传感器、光照强度传感器及控制大棚温湿度的大棚卷帘开关、 水泵开关、风扇开光等; Zigbee 协调器通过串口与Wifi 开发

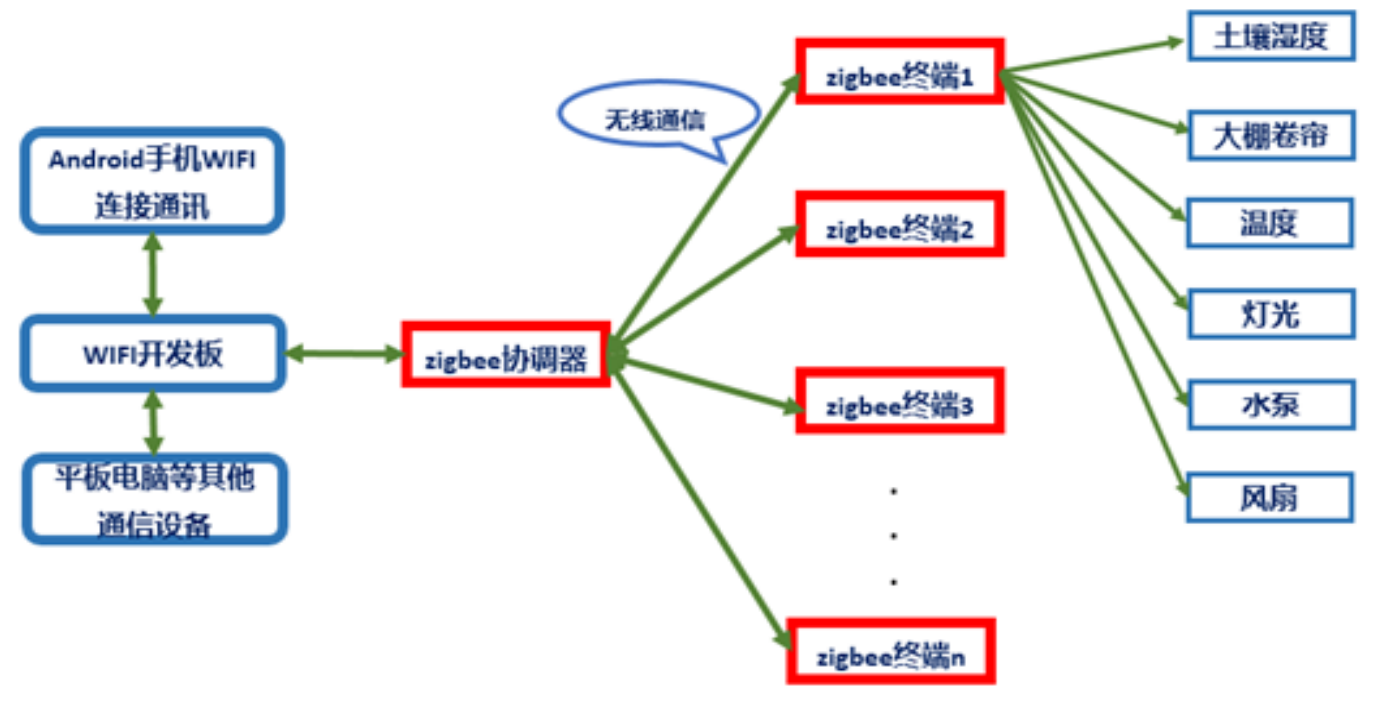

图 1 系统总体结构图

板进行通信, 将采集的数据传输给Wifi 开发板, Wifi 开发板接收来自 Android 控制端的信号, 并通过串 口发送给协调器; Android 终端控制模块安装有专门开发的监控程序, 通过 Wifi 与 Wifi 开发板进行通信, 可以在 Android 界面显示当前系统的实时环境信息, 并通过参数设置, 手动或者自动的对环境参数进行调 
节。

\section{2 系统硬件设计}

本系统的硬件主要有无线传感器终端模块、协调器模块、wifi 开发板模块等, 接下来给出 Zigbee 接 口电路, 温湿度传感器电路及土壤湿度传感器接口电路。

\section{2. $1 \mathrm{Zigbee}$ 接口电路的设计}

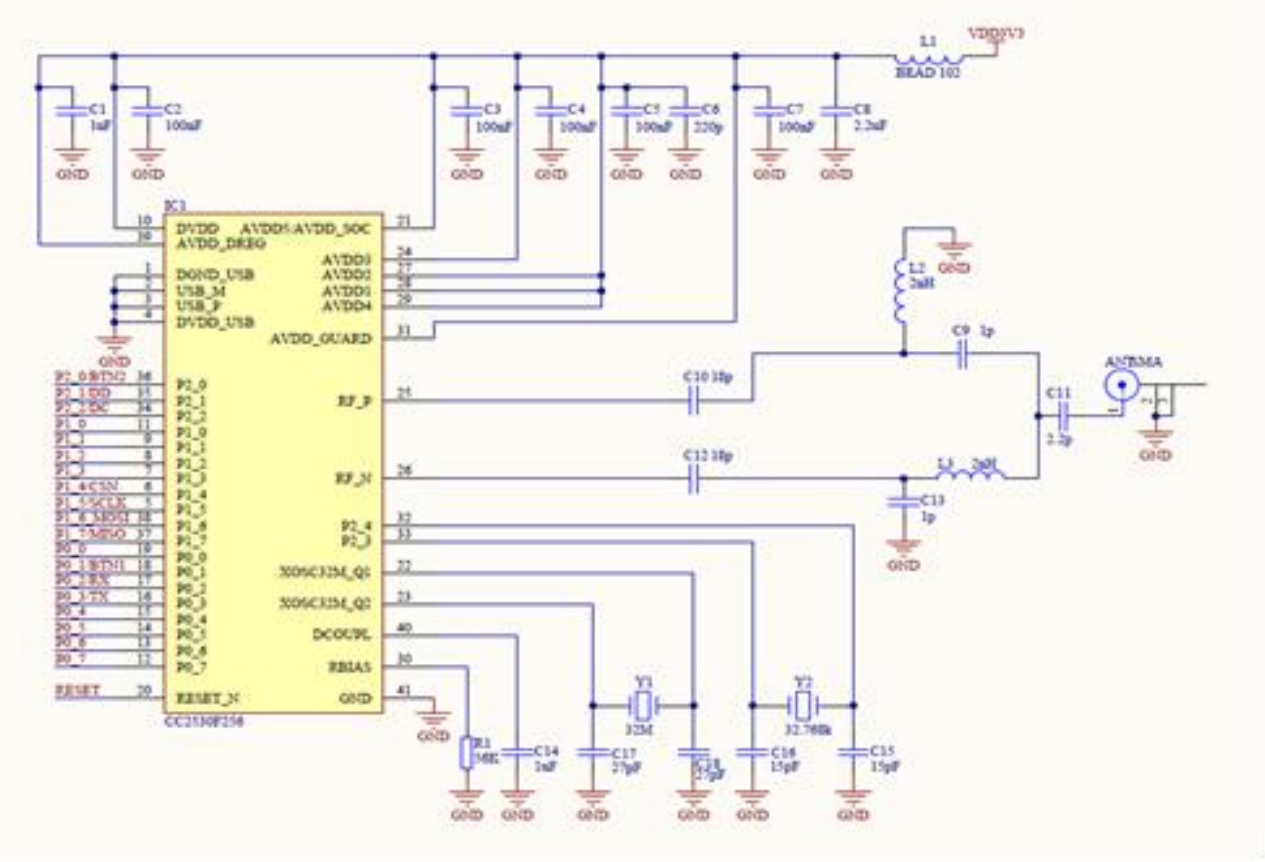

图 2 Zigbee 接口电路

Zigbee 接口电路如图 2 所示, 无线 Soc 采用 TI 公司的 CC2530F256 芯片, 该芯片支持最新的 Zigbee2007 协议栈, 该协议栈具有更好的互操作性、节点密度管理、数据负荷管理、频率捷变等方面有重大进步, 并 且支持网状网络, 而且其相对于以前的版本, 具有功耗低的优点。

\section{2 Wifi 接口电路的设计}

WiFi 模块采用全新的第三代嵌入式 Uart-WiFi 模块产品 HF-A $11 \times$ Uart-WiFi 是基于 Uart 接口的符 合 WiFi 无线网络标准的嵌入式模块, 通过 FCC, CE, RoHS 认证, 内置无线网络协议 IEEE802. 11 协议栈以 及 TCP/IP 协议栈, 支持频率范围: 2.412-2.484GHz, 能够实现用户串口或 TTL 电平数据到无线网络之间的 转换。处理器 S3C2440A 的串口直接与 HF-A11x 的串口相连, 进行通信, 电路设计连接如图 3 所示。

图 $3 \mathrm{WiFi}$ 接口电路图

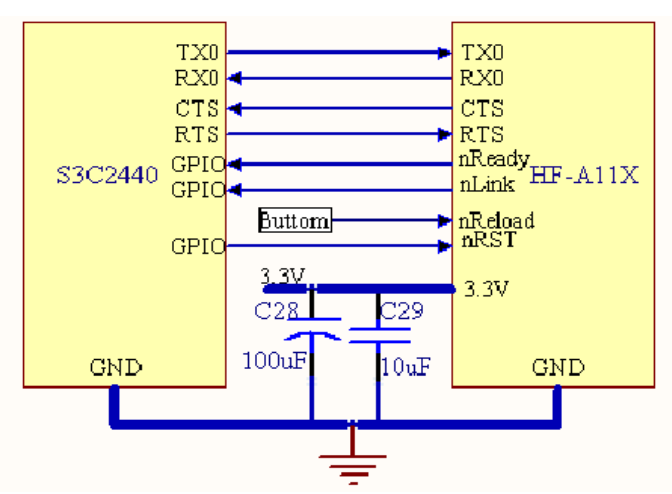




\section{3 土壤湿度传感器接口电路的设计}

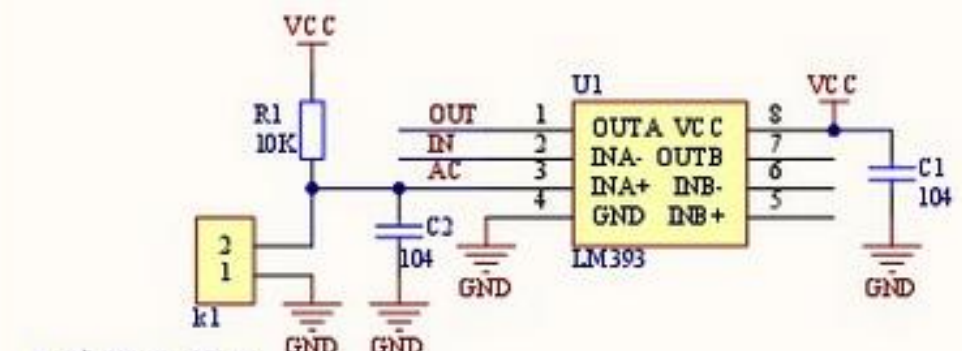

土壇探头接口

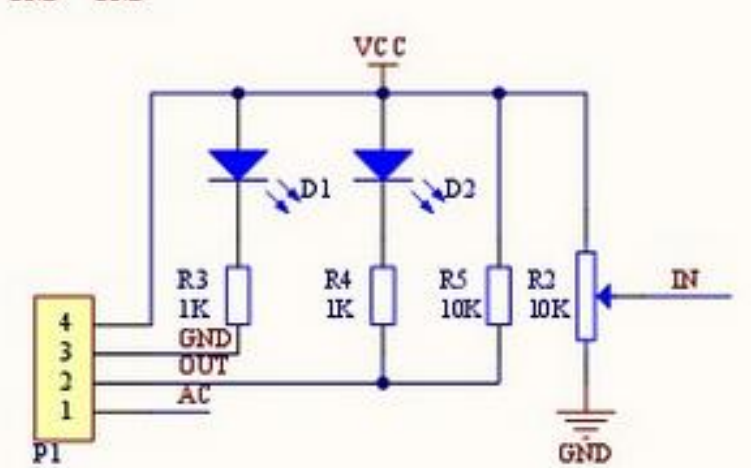

图 4 土壤湿度传感器接口电路设计图

土壤湿度传感器是由于土壤中含有矿物质离子，这些矿物质离子都溶解在土壤中的水中。以 FC-28 湿 度传感器作探头, LM393 做比较器, 两个电极插入土壤后充当 电阻, 与芯片中的电阻分压, 再送到 LM393 同相端中与变阻器得到的电压比较, 在土壤湿度达不到设定阈值时, D0 口输出高电平, 当土壤湿度超过设 定阈值时, D0 口输出低电平。电路设计如图 4 所示。

\section{3 系统软件设计}

系统软件主要包括以下几部分:

(1) 终端节点信息获取及控制: 基于 CC2530 芯片 (包括无线收发及传感器) 设计无线传感网络模块，并 完成各节点模块的软件编写和节点间通信调试;

(2) 协调器与 wifi 模块通信程序: 该部分程序实现 CC2530 协调器模块与 S3c2440 模块通过串口进行 数据交换;

(3) 以太网传输模块: 利用 S3c2440 控制网络接口芯片与 Android 设备通过Wifi 进行通信, 完成数据 的交换;

(4) 智能设备显示界面及监控程序: 利用 eclipse (Android 开发工具) 编写智能设备显示及控制界面程 序, 使经过数据处理模块处理后的数据通过无线网上传到智能设备界面上, 实现统一管理。

\section{4 结束语}

在基于 ZigBee 的智慧农业系统中，将无线网络技术应用于农业管理已成为势不可挡的趋势。无线网 络可以提供更大的灵活性、流动性, 省去花在综合布线上的费用和精力, 随着无线网络技术的进一步发展, 必将大大促进设备管理智能化的进程。此外, 本方案中采用了 Android 智能手机操作系统, 与其他手机操 作系统相比, Android 具有较好的开放性的特点。而且次系统通过无线通讯对各个终端的控制可以实时监 控和操作。在终端上留有其他扩展模块, 用户可以根据需要加入其他功能。比如在较为寒冷的地区, 可以 
增加加温等设备等。

\section{5 致谢}

榆林市科技局资助项目; 榆林学院教学改革研究项目。

\section{Acknowledgement}

Project supported by Yulin science and technology bureau; Yulin University teaching reform project.

\section{参考文献}

[1] 付玉志. 基于 ZigBee 技术的智慧农业实时采集和远程控制系统[D].浙江大学,2015: 14-16.

[2] 杨诚, 聂章龙. ZigBee 网络层协议的分析与设计[J]. 计算机应用与软件, 2009，26(12): 219-221.

[3] 覃梦甜. 基于物联网的智能农业系统运用[D].武汉轻工大学,2014: 5-8.

[4] 翟搯. 智能农业平台开发[D].浙江理工大学,2014: 13-17.

[5] 仇天月,陈旭,马超,袁涛,李琳一,赵京音. 基于 Android 智能手机的农业物联网信息采集和发布系统的研究[J]. 上海农业学 报,2014,02: 6-9.

[6] 任华,邹承俊. 基于物联网的智能农业系统研究与实现[J]. 物联网技术,2014,06:66-68.

[7] 管继刚. 物联网技术在智能农业中的应用[J]. 通信管理与技术,2010,03:24-27+42.

[8] 徐珍玉. “物联网+”现代农业发展新机遇[J]. 上海信息化,2015,06:22-24.

[9] Yong Tae Park, Jae-Young Pyun. Smart Digital Door Lock for the Home Automation[C]. 2009 IEEE Region 10 Conference, Singapore, 2009: 1-6.

[10] 㫿智雷,刘盛德,胡咸斌. ZigBee 技术及应用[M]. 北京:北京航空航天大学出版社,2007.

[11] 杨新华，王鹏，周仁伟. 基于 ZigBee 协议的远程自动抄表系统的设计[J]. 电气自动化，2009，31(5)：45-47.

\section{References}

[1] Zhi. Pay wisdom agriculture real-time data acquisition and remote control system based on ZigBee [D]. of Zhejiang University, 2015:14-16.(in Chinese)

[2] Yang Cheng, analysis and design of the.ZigBee network layer protocol [J]. computer applications and software, 2009, 26 (:219-221.) (in Chinese)

[3] Qin dream sweet. Intelligent agricultural system networking with [D]. of Wuhan University of light industry based on 2014:5-8. (in Chinese)

[4] Qu Tao. Intelligent agriculture platform [D]. of Zhejiang Sci-Tech University, 2014:13-17. (in Chinese)

[5] Chen Xu, Ma Chao, Qiu days to months, Yuan Tao, Li Lin, Zhao Jingyin. Agricultural IOT information collection and research of Android intelligent mobile phone publishing system based on 2014,02: [J]. Journal of Shanghai Agricultural University, 6-9. (in Chinese)

[6] Ren Hua, Zou Chengjun. Research and implementation of intelligent agriculture system based on Internet of things [J]. 2014,06:66-68. (in Chinese)

[7] Jigang. Networking technology in Intelligent Agriculture in management and application of [J]. communication technology, 2010,03:24-27+42. (in Chinese)

[8] Xu Zhenyu. "Internet of things +" new opportunities for modern agricultural development [J]. Shanghai informatization, 2015,06:22-24. (in Chinese)

[9] Yong Tae Park, Jae-Young Pyun. Smart Digital Door Lock for the Home Automation[C]. 2009 IEEE Region 10 Conference, Singapore, 2009: 1-6.

[10] Qu Minlei, Liu Shengde, Hu Xianbin. ZigBee technology and application [M]. Beijing: Beihang University press, 2007(in Chinese)

[11] Yang Xinhua. Design of remote automatic meter reading system based on ZigBee protocol [J]. automation of electric power, 2009, 31 (5):45-47. (in Chinese) 